\title{
Myositis ossificans-like soft tissue aneurysmal bone cyst: a clinical, radiological, and pathological study of seven cases with COL1A1- USP6 fusion and a novel ANGPTL2-USP6 fusion
}

\author{
Lingxin Zhang ${ }^{1,2} \cdot$ Sinchun Hwang ${ }^{3} \cdot$ Ryma Benayed $^{1} \cdot$ Guo Gord Zhu ${ }^{4} \cdot$ Kerry A. Mullaney ${ }^{1} \cdot$ Kelly M. Rios ${ }^{1}$. \\ Purvil Y. Sukhadia ${ }^{1}$. Narasimhan Agaram ${ }^{1}$ - Yanming Zhang ${ }^{1} \cdot$ Julia A. Bridge ${ }^{5,6} \cdot$ John H. Healey $^{7}$. \\ Edward A. Athanasian ${ }^{7} \cdot$ Meera Hameed $^{1}$
}

Received: 3 November 2019 / Revised: 21 February 2020 / Accepted: 23 February 2020 / Published online: 10 March 2020

(c) The Author(s), under exclusive licence to United States \& Canadian Academy of Pathology 2020

\begin{abstract}
Herein we described the clinical, radiological, histological, and molecular characteristics of seven soft tissue aneurysmal bone cysts (STABCs) diagnosed and managed at a tertiary cancer center and to elucidate their relationship with myositis ossificans (MO). All cases had established imaging and histopathological diagnosis of STABC and were subject to fluorescence in situ hybridization (FISH) for USP6 rearrangement and Archer ${ }^{\circledR}$ FusionPlex $^{\circledR}$ targeted RNA sequencing (RNASeq) analysis to identify the fusion partner. A thorough literature review of STABC and MO was conducted. The patients presented with painful masses unpreceded by trauma, occurring most commonly in the deep soft tissue of the thigh/ gluteus (4/7), and also in the supraclavicular region, the axilla, and the hand. On imaging, the lesions were frequently associated with peripheral calcification on conventional radiographs and CT (6/7), cystic components on ultrasound, as well as perilesional edema (7/7) and fluid levels (3/7) on MRI. Bone scan (1/1) showed intense radiotracer uptake. Histologically, 6/7 cases demonstrated zonal arrangements reminiscent of MO. USP6 rearrangement was found in all seven cases by FISH and/or RNASeq. RNASeq further detected COL1A1-USP6 fusion in six cases and a novel ANGPTL2-USP6 fusion in one case. Four patients underwent resection of the tumors and were disease free at their last follow-up. Three patients who underwent incisional or needle biopsies had no evidence of disease progression on imaging studies. In conclusion, the clinical, radiological, and pathological overlap between STABC and MO suggests that they are closely related entities. A novel fusion ANGPTL2-USP6 is associated with distinct clinical and pathological presentation.
\end{abstract}

Meera Hameed

hameedm@mskcc.org

1 Department of Pathology, Memorial Sloan Kettering Cancer Center, New York, NY 10065, USA

2 Department of Pathology and Laboratory Medicine, Hospital for Special Surgery, New York, NY 10021, USA

3 Department of Radiology, Memorial Sloan Kettering Cancer Center, New York, NY 10065, USA

4 Department of Pathology, Cooper University Health Care, Camden, NJ 08103, USA

5 Department of Pathology and Microbiology, University of Nebraska Medical Center, Omaha, NE 68198, USA

6 Division of Molecular Pathology, The Translational Genomics Research Institute, Phoenix, AZ 85004, USA

7 Department of Orthopedic Surgery, Memorial Sloan Kettering Cancer Center, New York, NY 10065, USA

\section{Introduction}

Although primary aneurysmal bone cyst (ABC) of bone was originally thought to be reactive when the entity was recognized by Jaffe and Lichtenstein in 1942 [1], evidence suggesting its neoplastic nature was established when Panoutsakopoulos et al. [2] discovered a recurrent chromosome aberration, $t(16 ; 17)(\mathrm{q} 22 ; \mathrm{p} 13)$. This was subsequently proven by Oliveira et al. to result in a fusion gene, in which the promoter region of the osteoblast cadherin 11 gene $(\mathrm{CDH11})$ at $16 \mathrm{q} 22$ is fused to the entire ubiquitin-specific protease 6 (USP6; alias Tre2) coding sequence at 17p13, transcriptionally upregulating the latter [3,4]. Alternative $5^{\prime}$ fusion partners to USP6 gene-THRAP3 (1p34.3), FOSL2 (2p23.2), CTNNB1 (3p22.1), CNBP (3q21.3), SEC31A (4q21.22), SPARC (5q33.1), OMD (9q22.31), PAFAH1B1 (17p13.3), COL1A1 (17q21.33), EIF1 (17q21.2), STAT3 (17q21.2), and USP9X (Xp11)—have been subsequently 
described [5-9], whereas $C D H 11$ remains the most common fusion partner in primary $\mathrm{ABCs}$ of bone.

Oliveira et al. tested for USP6 rearrangements in morphological mimickers of primary $\mathrm{ABCs}$, such as secondary $\mathrm{ABCs}$, myositis ossificans (MO), brown tumor, and cherubism, and found USP6 rearrangements in 2 of 12 cases with classic radiological and histological features of MO [10]. Recently, MO-like lesions with USP6 rearrangement were reported under both terms of MO [11, 12] and soft tissue aneurysmal bone cyst (STABC) [13]. The discordance in nomenclature in part reflects the overlapping radiological and pathological features of these newly defined USP6-rearranged lesions with $\mathrm{MO}$ and primary bone $\mathrm{ABC}$. Our cases herein referred to as MO-like STABCs emphasize this diagnostic challenge with detailed case examples and the value of molecular analysis for diagnostic confirmation. The objective of this study is twofold-(1) to describe the clinical course, radiological characteristics, and histological features of MO-like STABC; (2) and to identify the fusion partners to USP 6 by targeted RNA sequencing (RNASeq).

\section{Material and methods}

The study was conducted at a tertiary cancer center with approval by the Institutional Review Board (protocol\# 18-215).

Eight patients with MO-like STABCs diagnosed between 2009 and 2018 were identified by searching the pathology laboratory information system. Hematoxylin and Eosin-stained slides from archival formalin-fixed paraffin-embedded (FFPE) blocks were reviewed and radio-pathological correlation was performed. Specimens with sufficient tumor content $(>10 \%)$ that were not subjected to acid decalcification were submitted for molecular studies.

\section{Fluorescence in situ hybridization (FISH) analysis for USP6 rearrangement}

FFPE tissue samples were tested for USP6 rearrangements by a break-apart FISH assay using a commercial USP6 break-apart FISH probe (Zytovision, 27572 Bremerhaven, Germany). FFPE tissue sections (4 mm thick) generated from FFPE blocks of tumor specimens were pretreated by deparaffinization in xylene and dehydration in ethanol. The dual-color FISH assay was conducted according to the protocol for FFPE sections from Vysis/Abbott Molecular with a few modifications. FISH analysis and signal capture were conducted on a fluorescence microscope (Zeiss, Jena, Germany) coupled with the ISIS FISH Imaging System
(Metasystems, Altlussheim, Germany). We analyzed 100 interphase nuclei from each tumor specimen, except for case 7 , in which 50 interphase nuclei were analyzed due to regional suboptimal signal quality.

\section{RNA-extraction and -targeted sequencing}

A minimum of ten unstained slides were subject to deparaffinization. RNA extraction was then performed by using RNeasy FFPE Kit according to the manufacturer's protocol (Qiagen, Catalog \#73504). cDNA libraries were constructed according to the Archer FusionPlex ${ }^{\mathrm{TM}}$ protocol (previously described by Zheng et al. [14]) with manufacturer supplied reagents, including Archer $^{\circledR}$ Universal RNA Reagent Kit for

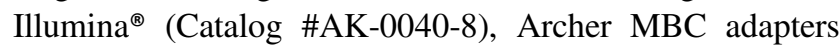
(Catalog \#SA0040-45), and custom designed gene-specific primer (GSP) pool kit. A total 346 fusion unidirectional GSPs, ranging in length from 18 to 39 base pairs, have been designed to target-specific exons in 62 genes known to undergo rearrangement in tumorigenesis based on the current literature [15]. GSPs are hybridized in either $5^{\prime}-3^{\prime}$ or $3^{\prime}-5^{\prime}$ direction to the relevant exons of each gene in order to enrich the cDNA libraries for known and novel fusion transcripts in combination with adapter-specific primers. The enriched amplicons were sequenced on an Illumina MiSeq instrument. A high confidence fusion event was reported when supported by a minimum of five breakpointspanning unique reads and three breakpoint-spanning reads with unique start sites.

\section{Results}

\section{Clinical, radiological, and pathological features}

Of the eight MO-like STABCs, one case showed USP6 rearrangement by FISH but was excluded from the study due to insufficient material for RNASeq.

The remaining seven patients included five females and two males with a median age of 32 years (14-51 years). All patients presented with a painful mass without antecedent trauma. Two patients (cases 3 and 5) had symptoms for more than 6 months. These lesions ranged from $2.4 \mathrm{~cm}$ to $6.0 \mathrm{~cm}$ in the greatest dimension and were located in the thigh/gluteus (4), supraclavicular region (1), axilla (1), and hand (1). Two lesions were juxtacortical (cases 2 and 6). All patients underwent imaging studies. Four lesions were solid (4/7), while others were partially cystic with fluid levels (3/7) on ultrasound and/or MRI. Peripheral calcifications were typically seen on plain radiograph and CT (5/ 7) at initial presentation, which increased over the course. On MRI, the lesions exhibited perilesional edema (7/7) with enhancement. Bone scan (1/1) showed intense 

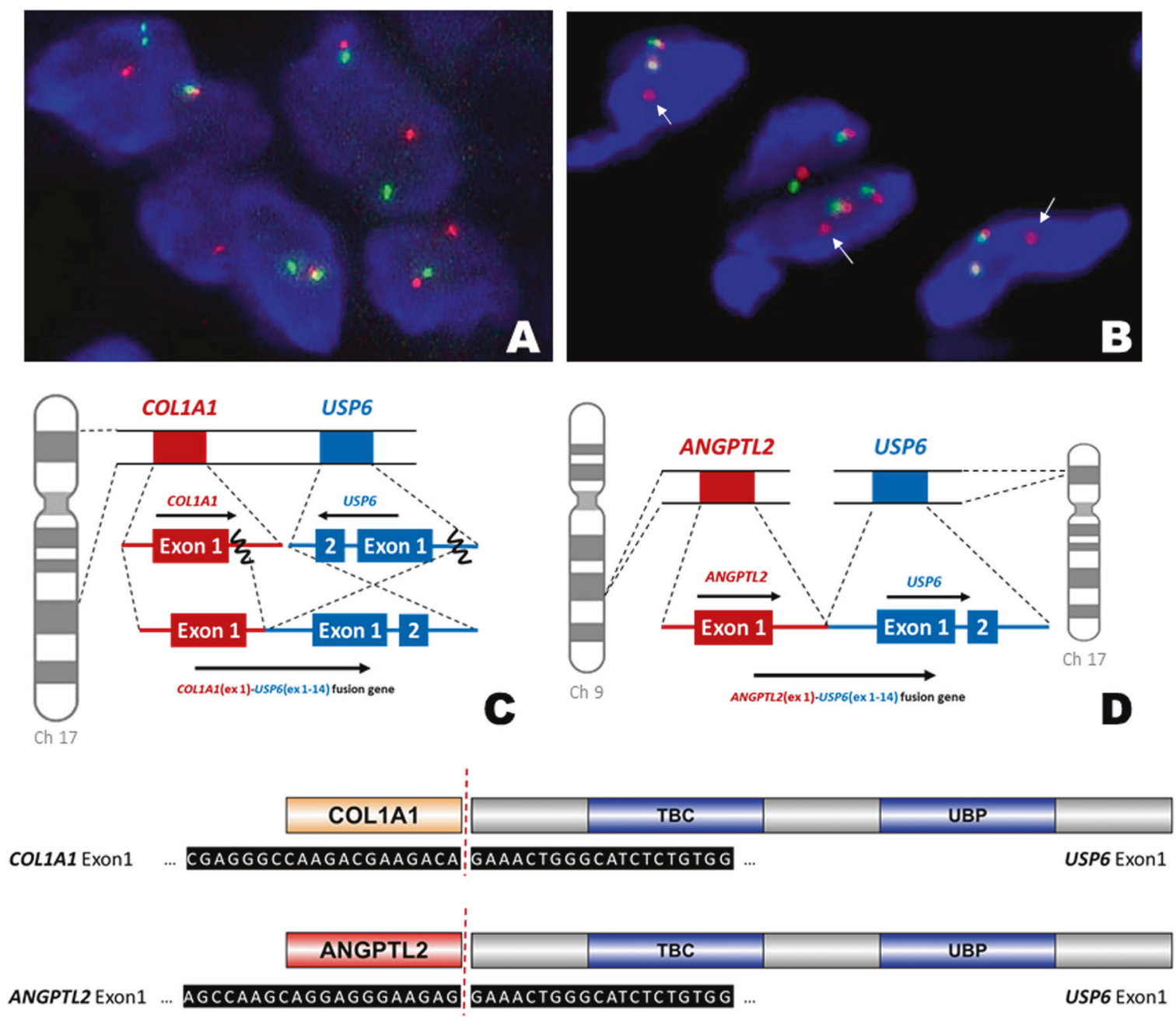

를

Fig. 1 Fusion patterns in MO-like STABCs. a Fluorescence in situ hybridization (FISH) analysis with break-apart probes flanking the USP6 gene $\left(5^{\prime}\right.$ - green, $3^{\prime}$-orange) showed a split of the green and orange signals, indicating disruption of USP6. b Two normal signals and a single $3^{\prime}$ (arrow) signal indicative of USP6 gene rearrangement was seen in case 3 . c This schematic diagram represents the most

radiotracer uptake, which markedly decreased at a followup bone scan. Histologically, 6/7 cases (except for case 3) had zonal growth pattern seen in classic MO, composed of (myo)fibroblastic spindle cells, multinucleated giant cells, and blood-filled sinusoids in the center, in most cases confined by reactive woven bone at the periphery, while the lesion in case 3 was infiltrative with clustered calcification. The FISH study in 6/7 cases showed rearrangement of USP 6 with either split of the $5^{\prime}$ and $3^{\prime}$ signals or a single $3^{\prime}$ signal. Subsequent RNASeq detected USP6 rearrangement in all seven cases. In 6/7 cases RNASeq showed a COL1A1-USP6 fusion. Case 3 had a novel ANGPTL2USP6 fusion. In all cases, the breakpoints were located in exon 1 of USP6, placing the entire coding sequence of USP6 under the influence of the promoter region of common fusion pattern in our series of MO-like STABCs (6/7 cases), with breakpoints within exon 1 of COL1A1 (17q21-1722) and exon 1 of USP6 (17p13). d A novel fusion between ANGPTL2 (9q33) and USP6 was found in case 3. e The COL1A1-USP6 and ANGPTL2USP6 fusion products included the promoter region of the COL1A1 and ANGPTL2 and the entire coding sequence of USP6.

COL1A1 and most likely promoter region of ANGPTL2 in case 3 (see Fig. 1). Six patients (except for patient 4) were followed for a median of 26.5 months. Patient 3 underwent resection after having persistent symptoms for about 3.5 years. The remaining patients had resolved symptoms following resection or biopsy. The clinical, radiological, and pathological findings of all cases are summarized in detail in Table 1.

\section{Case 1}

A 32-year-old woman presented with an enlarging, painful mass in her left proximal thigh for 2 months (Fig. 2). Imaging studies showed a partially cystic mass without calcification. Although the clinical and imaging presentations were 


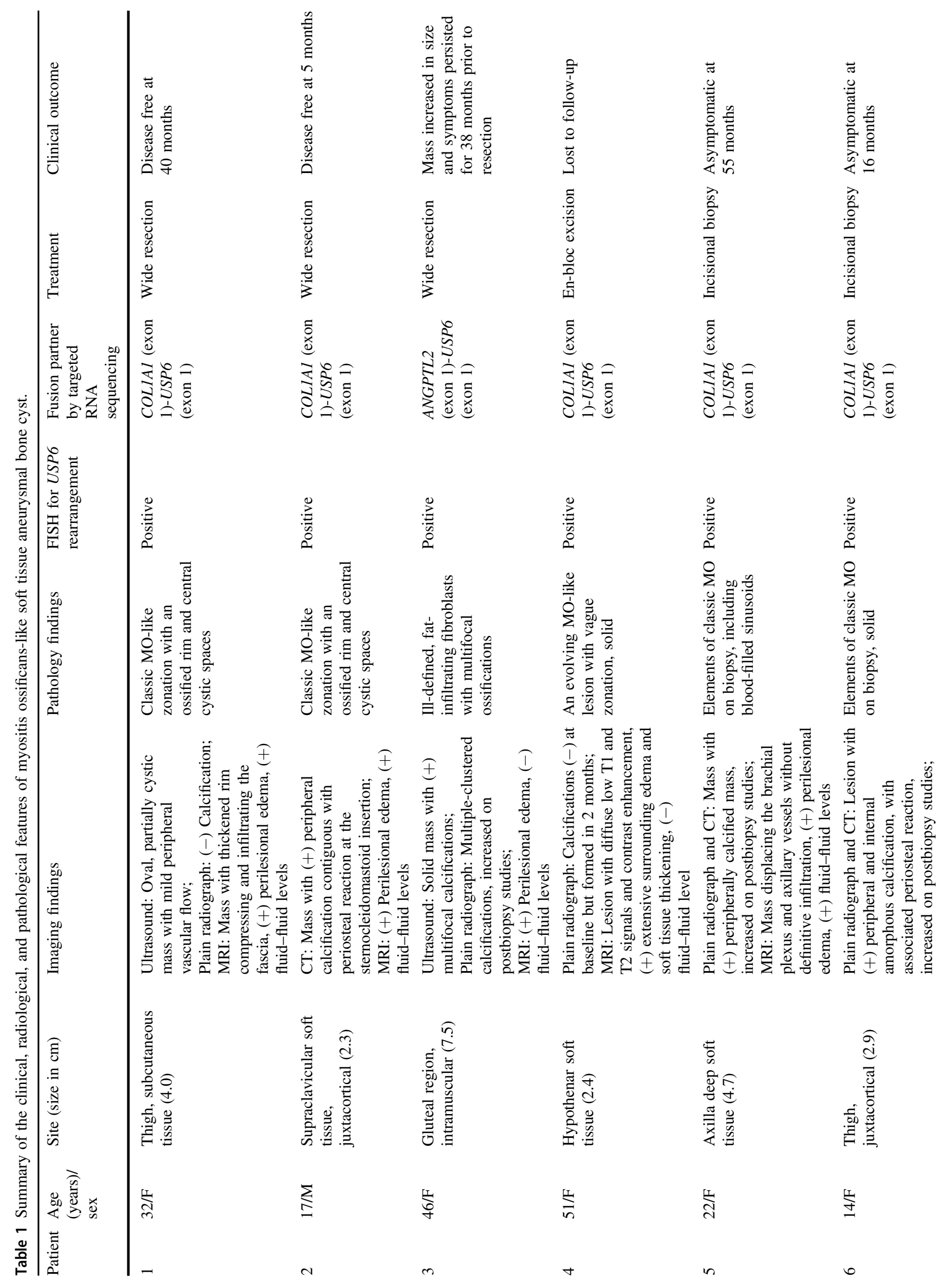




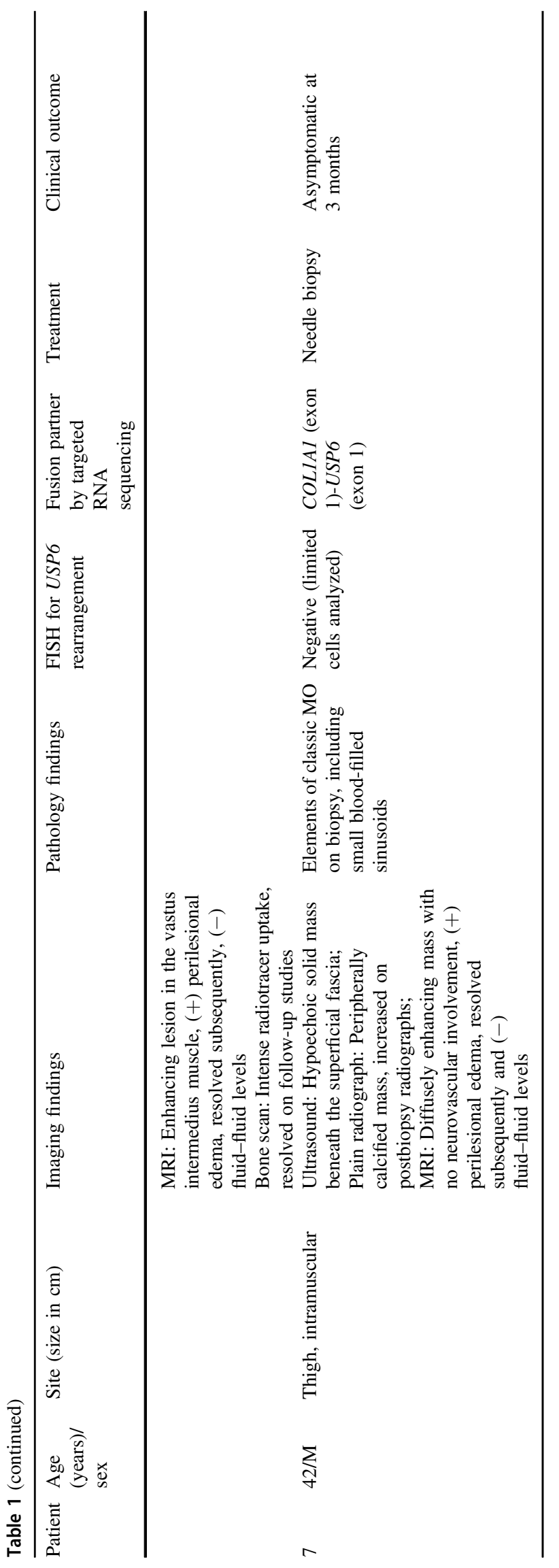

suggestive of a nodular fasciitis, the clinical course was not typical. A wide resection of the mass was performed. Grossly, the mass had a thick, ossified wall, and a central cystic space with hemorrhage. Microscopic examination showed a lesion with zonal phenomenon. The inner cystic areas were lined by fibroblast-like spindle cells, occasional osteoclast-like giant cells, and background inflammatory cells, reminiscent of $\mathrm{ABC}$. In the middle zone, osteoid was being laid down by the proliferating spindle cells, which gradually merged into the outer zone of trabeculated, osteoblast-rimmed woven bone, focally containing cartilage. The patient remained disease free 3.5 years postoperatively.

\section{Case 2}

A 17-year-old young man noted a painful, palpable mass in his left clavicle for 6 weeks. The radiological differential diagnoses included infection, intracortical osteoid osteoma, periosteal osteoblastoma, and STABC. The lesion was resected along with the periosteum. The macro- and microscopic findings were similar to those in case 1 . There was no recurrence 5 months after the resection.

\section{Case 3}

A 46-year-old woman noticed a mass in her left gluteus/ thigh that caused soreness (Fig. 3). On imaging studies, the mass continued to grow over 6 months from $5.1 \mathrm{~cm}$ to 6.0 $\mathrm{cm}$. Clinical and radiological differential diagnoses included deep fibromatosis (desmoid tumor), nodular fasciitis, and MO. Biopsy showed fibroblastic spindle cell proliferation in a collagenized stroma. The cellularity was variable but in general low. Osteoclast-like giant cells were focally scattered around osteoid. No blood-filled cysts or reactive bone trabeculae were seen. The mass was resected 3.5 years after the initial presentation given the patient's persistent symptoms. Gross examination showed a $7.5 \times 4.2 \times 2.9 \mathrm{~cm}$ predominantly solid, ill-defined, and lobulated mass with multifocal ossification involving the deep adipose tissue and skeletal muscle. Histologic sections showed fibroblastic proliferation that was previously seen on the biopsy infiltrating into adipose tissue with multiple haphazard foci of reactive woven bone.

\section{Case 4}

A 51-year-old woman suddenly noticed a tender lump in the hypothenar region of the left hand. A needle biopsy showed a solid fibroblastic proliferation in a loose stroma alternating with dense collagen bundles (osteoid), reminiscent of an early MO. After 3 months, the mass was extricated displaying a vague zonal pattern with markedly decreased 
Fig. 2 Case 1. A 32-year-old woman with a thigh mass status post resection. a $\mathrm{A}$ longitudinal ultrasound image of the anterior thigh showed a partially cystic subcutaneous mass with irregular rim and peripheral vascular flow. b On axial T2-weighted MR image, the mass had high signal with thickened rim and fluid level (arrowhead). It compressed on and infiltrated the adjacent fascia (arrows). c The excised mass had a tan-white, glistening, and firm cut surface with a central cystic space with hemorrhage. d The lesion displayed a MOlike zonal pattern composed of an outer shell of reactive woven bone with a hypocellular stroma (e) and central blood-filled cysts lined by fibroblast-like spindle cells, osteoclast-like giant cells, and background inflammatory cells, resembling primary $\mathrm{ABC}$ of bone (f).
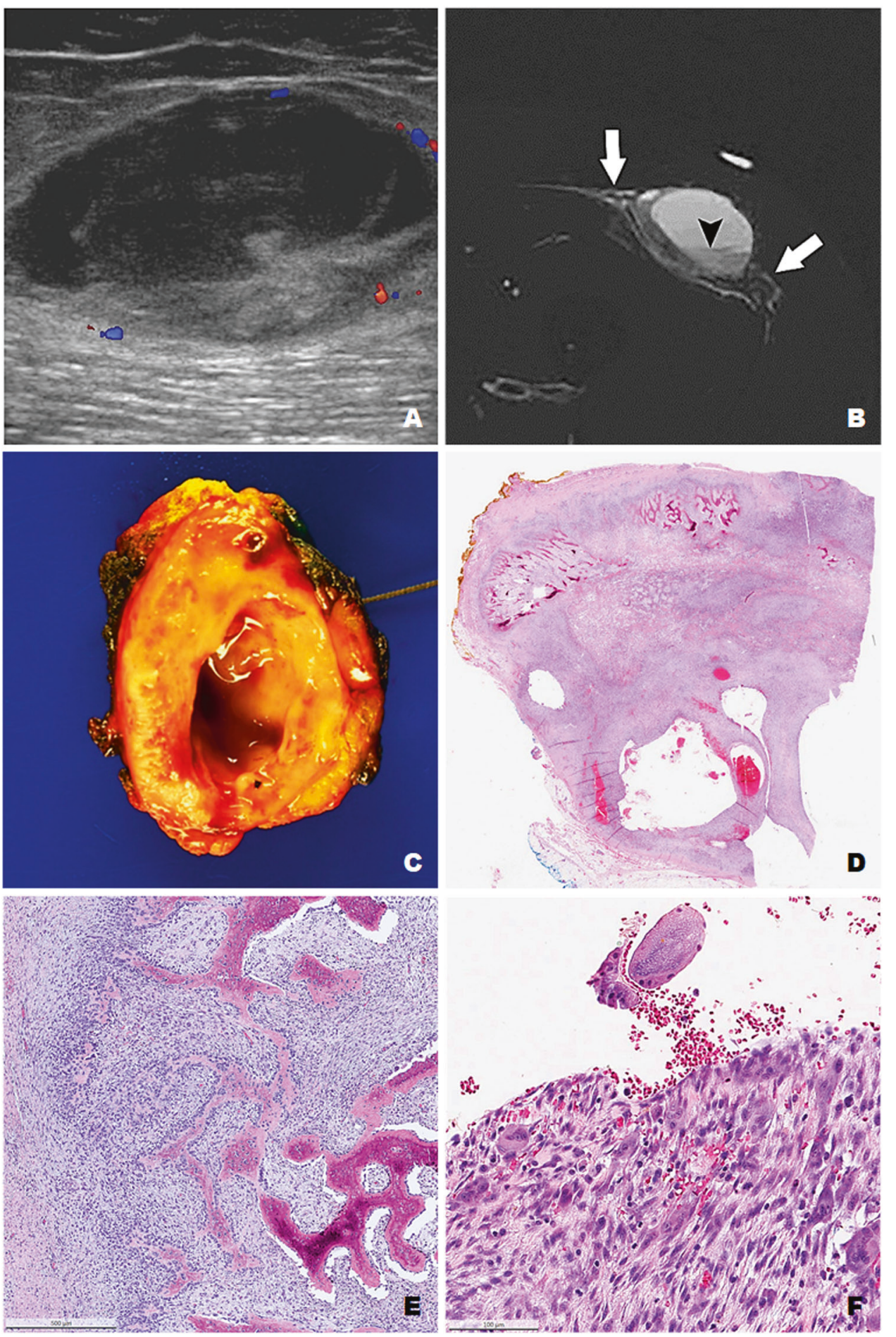

cellularity in the center and mineralized collagen matrix at the periphery. The patient was lost to follow-up.

\section{Case 5}

A 22-year-old woman presented with left shoulder pain for 6 months. Although the main consideration radiologically was a MO, the anatomic site, and protracted clinical course were rather unusual.

An open biopsy showed elements reminiscent of a MO, including fibroblastic spindle cell proliferation, osteoclast-like giant cells centered around blood-filled sinusoids, and osteoblast-rimmed woven bone maturing into lamellar bone. A resection was not performed given 
Fig. 3 Case 3. A 46-year-old woman with a gluteal/thigh mass status post resection (ANGPTL2-USP6 fusion). a A lateral radiograph showed a mass with clustered peripheral calcification (arrows) in the left proximal posterior thigh. $\mathbf{b}$ Axial T1-weighted and c T2weighted MR image showed a mass infiltrating the gluteus maximus and hamstring muscles. The mass consisted of an ossified component (arrow) and a nonossified soft tissue component (curved arrows). d A needle biopsy showed solid fibroblastic spindle cell proliferation in a collagenized stroma. e Gross examination showed an ill-defined and lobulated mass with multifocal ossification involving the deep adipose tissue and skeletal muscle. f Both ossified and nonossified components were present on histologic examination. In contrast to other cases with a zonal pattern, the nonossified component composed of spindle cells infiltrated the adipose tissue with multiple haphazard foci of woven bone.
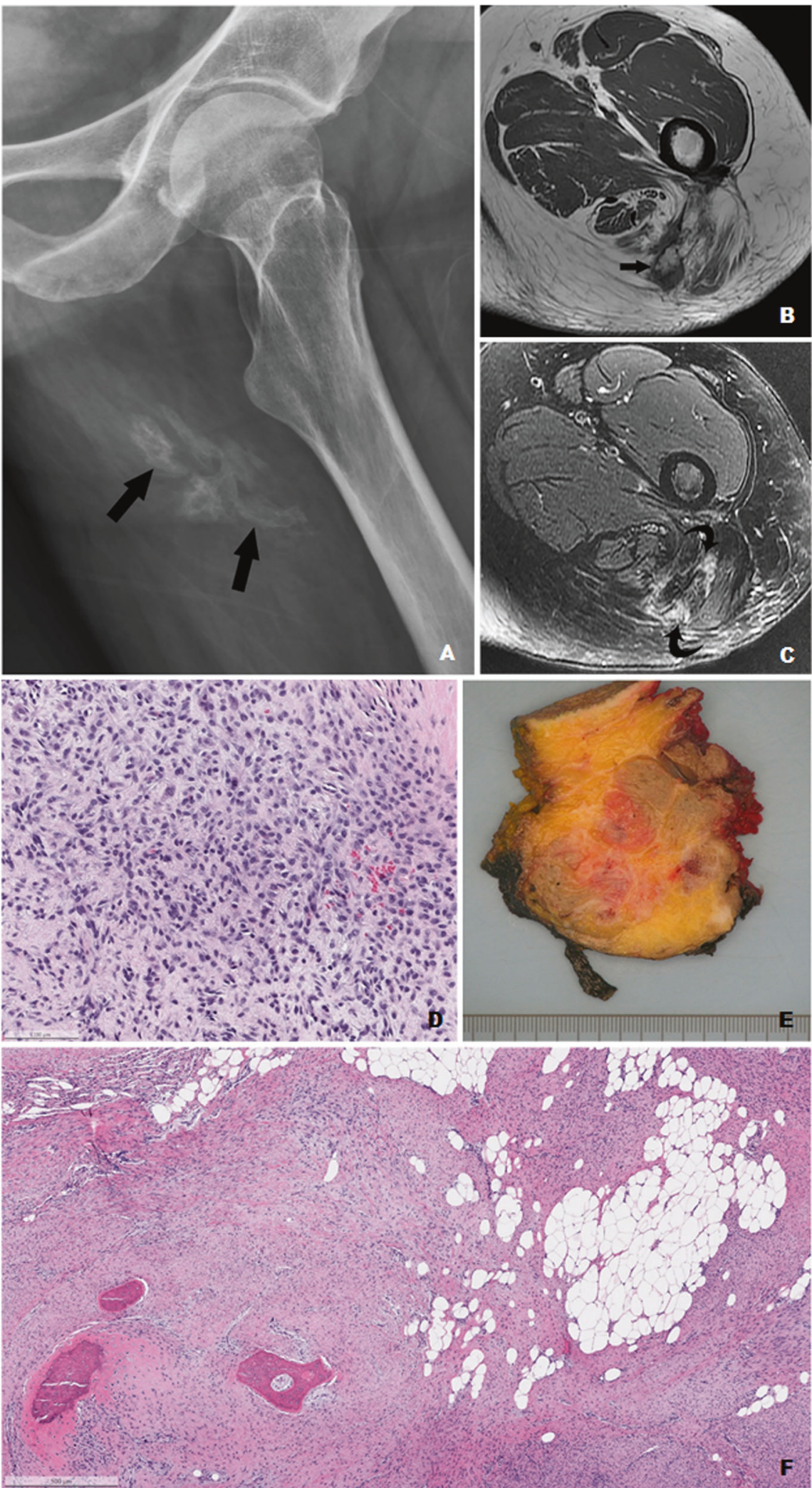

the proximity of the lesion to the brachial plexus and axillary vessels. The patient reported significantly improved range of motion after the open biopsy, and her axillary mass had decreased in size and residual dense calcification and remained stable at 4.5 -year postoperative follow-up. 
Fig. 4 Case 6. A 14-year-old girl with a thigh mass status post incisional biopsy. a $\mathrm{A}$ radiograph showed a juxtacortical mass with calcifications (arrowheads) and periosteal reaction (arrows). b On axial MRI STIR image, the mass ( $\mathrm{T}$ ) was surrounded by edema (arrowheads) and thickened soft tissue in the vastus intermedius muscle. No fluid level was identified. c Axial T2-weighted fatsuppressed image of the followup MRI 10 months after the open biopsy showed unchanged thick periosteal reaction along the femoral cortex with almost complete resolution of the previously identified the high T2 signal, consistent with continued internal healing. d An incisional biopsy showed exuberant interconnecting woven bone formation and e neighboring areas of fibroblastic proliferation with small foci of osteoid matrix.
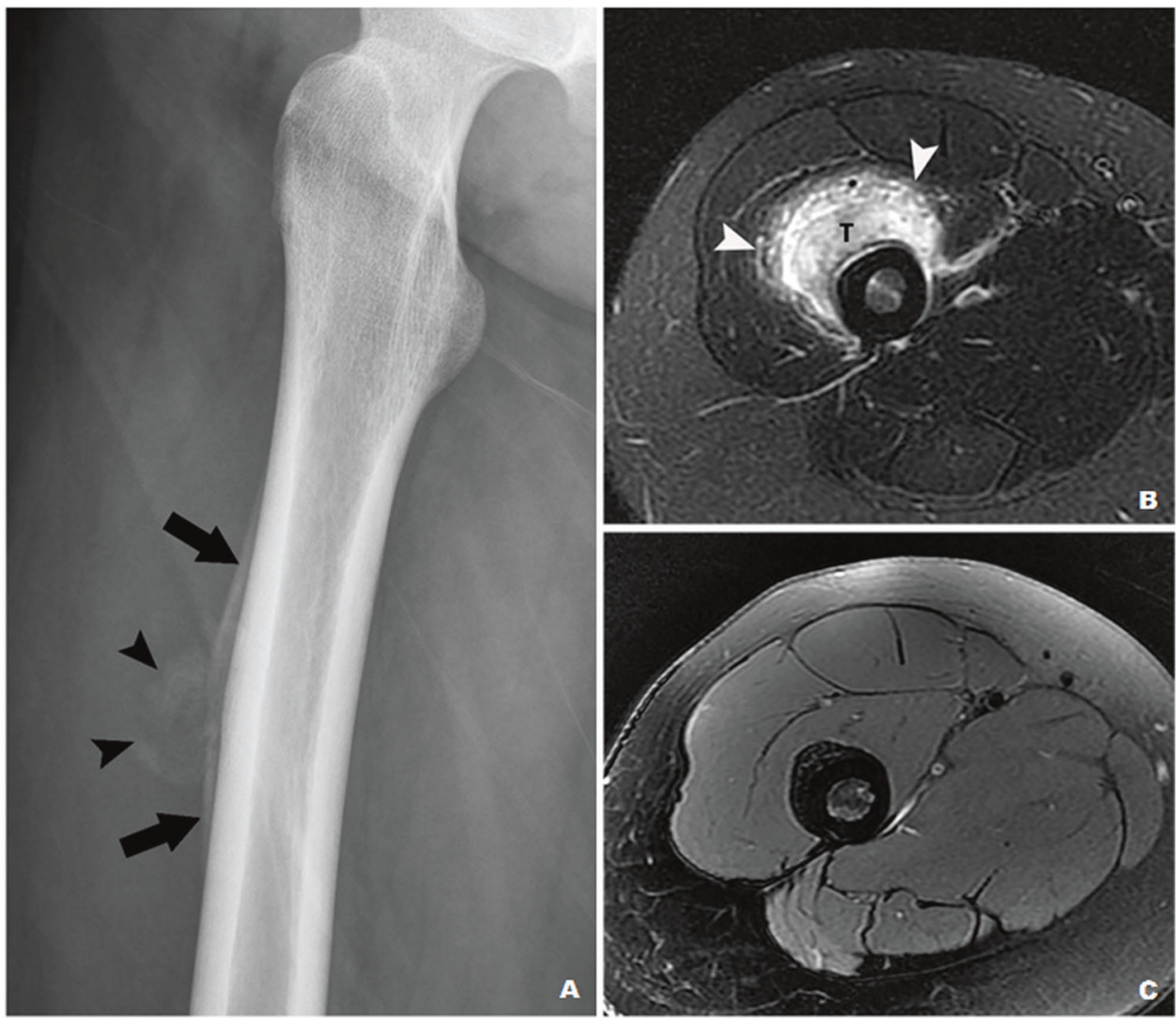

A
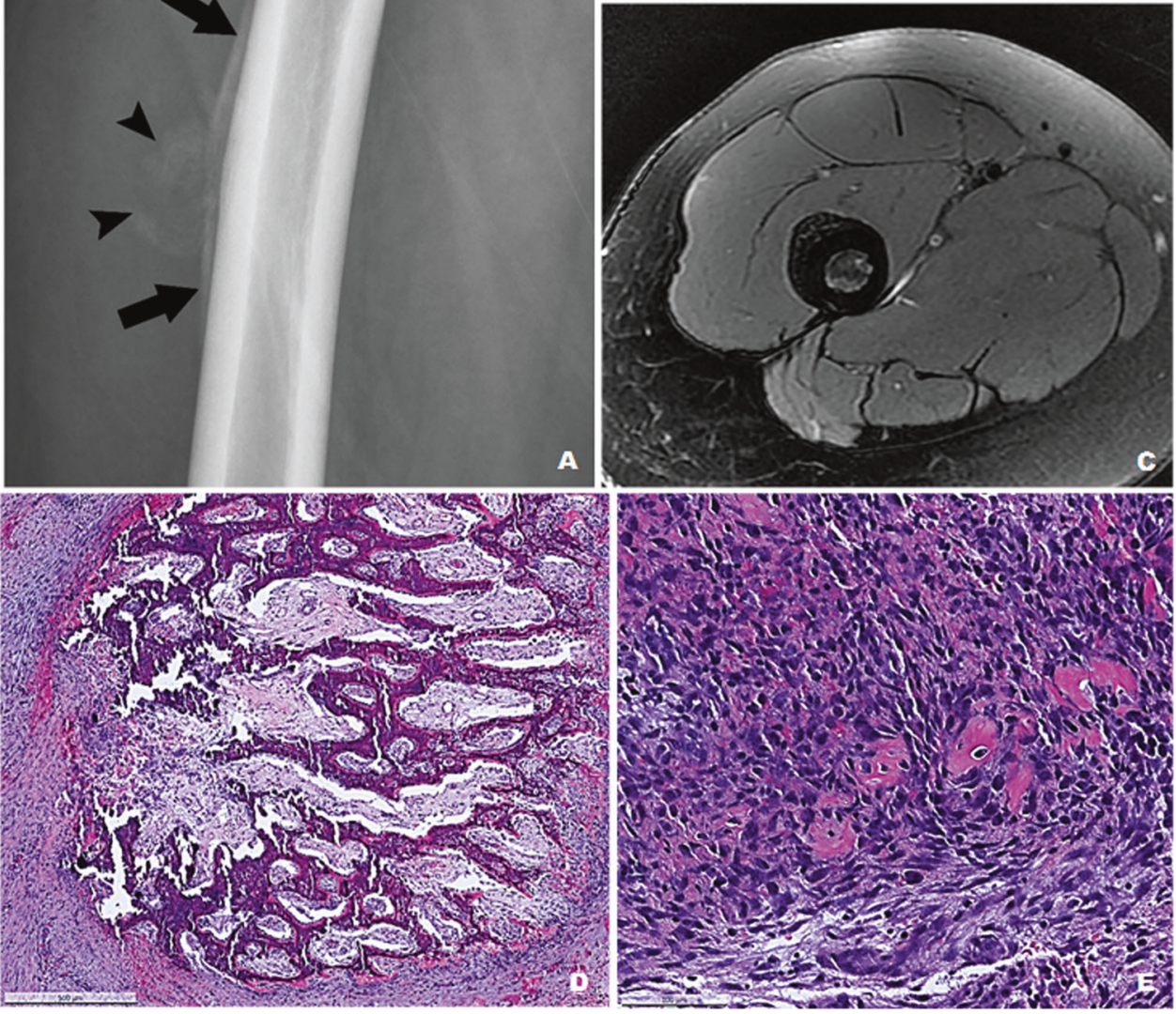

\section{Case 6}

A 14-year-old girl, an avid dancer, developed sudden pain in her right thigh 3 weeks prior to presentation (Fig. 4). The pain limited her mobility and was inadequately relieved by antiinflammatory medication. Imaging studies showed a solid, calcified lesion in the vastus intermedius muscle, with associated right femoral diaphyseal periosteal reaction and intense radiotracer uptake, raising concern for a surface osteosarcoma. No intracortical lesion was identified. Radiological differential diagnoses were broad, including solid $\mathrm{ABC}$, bizarre parosteal osteochondromatous proliferation, and MO. Bone tumors such as Ewing sarcoma, osteosarcoma, or osteoblastoma were also considered. An incisional biopsy showed a solid lesion with exuberant interconnecting woven bone, favoring a benign etiology. The bony matrix was embedded within a collagenized to myxoid stroma with variably cellular fibroblastic spindle cells and thick-walled vessels. Occasional osteoclast-like giant cells were also identified, however, without associated blood-filled sinusoids. The patient was asymptomatic at the last follow-up.

\section{Case 7}

A 42-year-old man developed subacute right thigh pain and swelling. Main differential diagnoses based on imaging 
findings included $\mathrm{MO}$ and solid $\mathrm{ABC}$. A needle biopsy demonstrated similar findings as those in case 5. In 3 months, the mass decreased in size and the patient's associated pain resolved.

\section{Discussion}

Historically, "myositis ossificans", also known as MO circumscripta and MO traumatica, is localized and selflimiting. The condition affects individuals of any age with a male predominance but is most commonly documented in adolescents and athletically active young adults. It is commonly agreed that trauma (e.g., stab wound, blunt trauma) or repetitive mechanical stress (e.g., "rider's bone", "fencer's bone") is the initiating event in most cases, while arthroplasty, fractures, spinal cord damage, and thermal injury are also risk factors $[16,17]$. It usually presents as a localized mass, but occasionally can be infiltrative. Within a few days of trauma, radiographs show a rounded discrete soft tissue swelling. It takes about 6 weeks before ossifications at the periphery becomes apparent on radiography. In about 2 months, MO reaches its maximal size and definitive outline, then continues to mature and ossify in 5-6 months. Although the condition is not rare, the pathogenesis of MO has not been well elucidated. Earlier authors placed $\mathrm{MO}$ in a middle ground between inflammatory process and a neoplasm, while some pathologists favor it to be a true neoplasm $[16,18,19]$. Undoubtedly MO is a benign process and may involute over time, therefore, can be conservatively managed. But it may present to the pathologist as a pseudosarcomatous soft tissue tumor, particularly during its rapidly developing phase and when it arises in the juxtacortical location, radiographically mimicking osteochondroma, or osteosarcoma (as in case 6). If biopsied, the histology may display a highly cellular osteoblastic proliferation with osteoid or woven bone formation. However, MO has a characteristic "zonal phenomenon," composed of a well-delimited outer rim of bony spicules, a central area of proliferating fibroblastic spindle cells with multinuclear giant cells, and a transition zone of osteoblasts with islands of immature bone. The circumscription with zonation usually separates $\mathrm{MO}$ from the more ominous infiltrating surface osteosarcomas however deceptively benign the cytology the latter may show.

In 1992, Amir et al. [20] reported a MO that spontaneously occurred in a 15-year-old girl, which contained a central radiolucent area made up of multiple blood-filled, cyst-like spaces. This lesion was interpreted as MO with ABC changes. Following this report, Rodríguez-Peralto et al. [21] first used the term "primary aneurysmal cyst of soft tissues" in describing a MO-like lesion with histological features identical to those found in $\mathrm{ABC}$ of bone.
More STABCs were described in the literature and almost all of them had overlapping clinical and radiological features of MO [10, 12, 13, 22-31] (Table 2). As with MO, STABC affects patients with a wide age distribution (6-62, mean age 30 years) without gender predilection and presents as a painful, rapidly enlarging mass. The thigh, gluteus, upper arm, shoulder, and axilla are most commonly affected regions. Although in previous reports cystic components were common on imaging studies [13, 25], fluid-fluid levels on MRI were only present in 3/7 (43\%) of our cases, which may be explained by different temporal stages. In the current standard care for MO-like lesions, a biopsy is only performed when the clinical presentation deviates from a classic MO, making watchful waiting clinically untenable. Thus there is a selection bias in STABC and, therefore, suggested clinical and radiological features to differentiate STABC from MO such as lack of perceived trauma and protracted clinical course are not entirely specific.

FISH and RNASeq studies for USP6 (chromosome 17p13.2) rearrangement in STABC [5, 10, 13, 27, 28, 31] have proved to be objective tools for diagnosis. The lesions in our series encompassed a variety of anatomic sites (e.g., axilla, tendon insertion site), beyond the intramuscular locations of classic MO. Still, the overlapping clinical, radiological, and pathological features between STABCs and MOs suggest that they are closely related entities. Indeed, Bekers et al. stated that MO is another "transient neoplasm" characterized by recurrent rearrangement of USP6, a promiscuous fusion partner identified in primary $\mathrm{ABC}$ of bone, nodular fasciitis [32, 33], cellular fibroma of tendon sheath [34], giant cell rich lesions of the small bones [35], and more recently in cranial fasciitis [36, 37] and fibro-osseous pseudotumor of digits [12, 38] (Table 3).

While primary $\mathrm{ABC}$ of bone demonstrates variant translocations with multiple partners, the fusion partners for STABC have remained relatively consistent with COL1A1 [5, 13, 31]. COL1A1 encodes for the collagen alpha 1 polypeptide chain and mutations in the gene are associated with many inherited disorders, such as osteogenesis imperfecta, Ehlers-Danlos syndrome, infantile cortical hyperostosis, and idiopathic osteoporosis [39]. In the neoplastic setting, this gene is well known for this diagnostic fusion with the platelet-derived growth factor B-chain $(P D G F B)$ gene in dermatofibrosarcoma protuberans and giant cell fibroblastoma driving the constitutive activation of $P D G F B$ gene [40]. COL1A1 is mapped to $17 \mathrm{q} 21-17 \mathrm{q} 22$, with opposite orientation with USP6 at $17 \mathrm{p} 13$. Previous cytogenetic with subsequent RNA sequencing studies $[25,31]$ in two STABCs showed COL1A1-USP6 fusion involving exon 1 of COLIA1 and a splicing variant of USP6 exon 1 or exon 2. The RNASeq results in six of our cases showed consistent breakpoints in exon 1 of COL1AI and USP6 exon 1. 


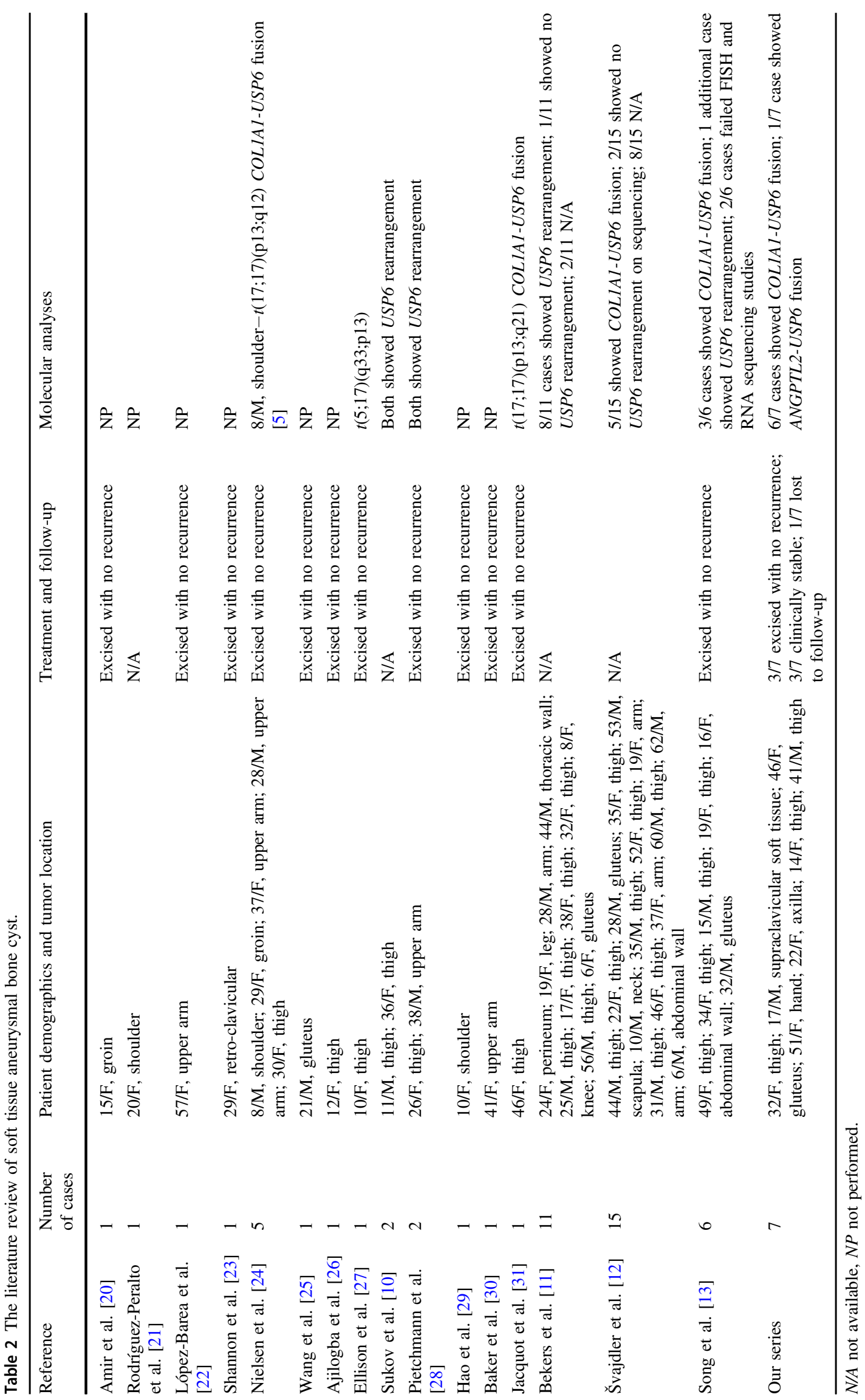




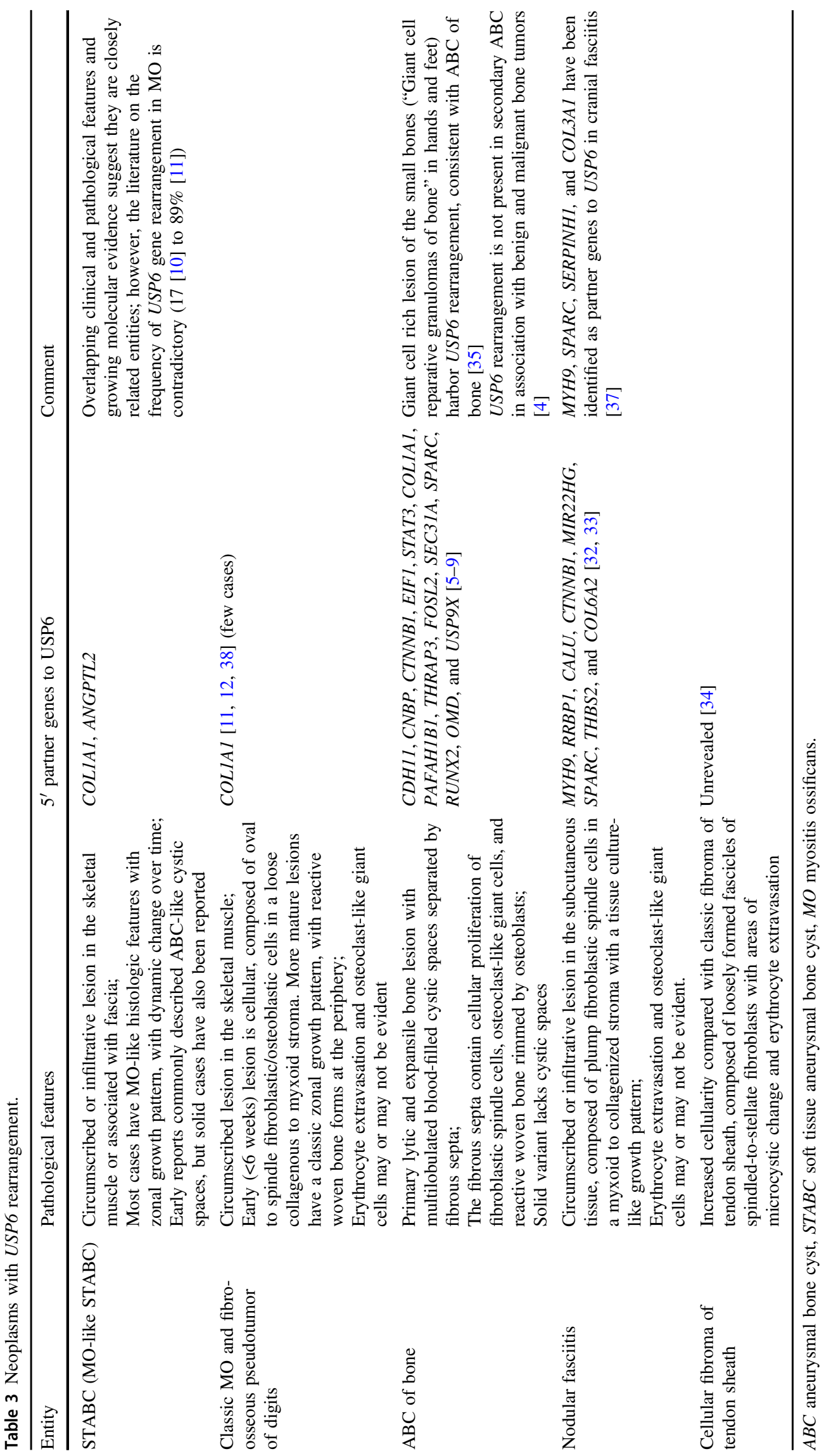


Case 3 was unique for the lack of zonal growth pattern and a novel ANGPTL2-USP6 fusion. ANGPTL2 (angiopoietin-related protein-2) at $9 \mathrm{q} 33$ is a member of vascular endothelial growth factor family, known not only for its angiogenic and antiapoptotic properties but also for its proinflammatory properties [41]. In our case, the fusion involved exon 1 of $A N G P T L 2$ and the entire coding sequence of USP6. It is likely that ANGPTL2 also contributes with its promoter. It is unknown how these fusions recapitulate similar pathological processes within bone and soft tissue.

Oliveira et al. [42] studied the molecular pathogenesis underlying the role of USP6 in ABCs and showed that USP6 residing in the fibrous stromal component of $\mathrm{ABC}$ induces transcription of matrix metalloproteinase through activation of the nuclear factor $\mathrm{\kappa B}(\mathrm{NF \kappa B})$, which seems to play a role in matrix degradation, angiogenesis, and inflammatory response. More recently, an essential mediator of USP6's pathogenic mechanism has been recognized as the Jakl-STAT3 pathway [43], which acts in concert with NFkB. This biological effect explains the histological features of the lesion mimicking a reparative/reactive hemorrhagic process and thus the long-term assumption that $\mathrm{ABC}$ was nonneoplastic albeit this was a destructive lesion of the bone. While the cell line of differentiation remains elusive, most of the reported fusion partners of $\mathrm{ABC}$ of bone are expressed during osteoblastic differentiation and it is suggested that the neoplastic cell could be derived from osteoblasts [5]. A common breakpoint is in exon 1 or 2 of the $5^{\prime}$ partner gene, which fuses with the first coding exon of USP6; the expected functional consequence being upregulated expression of an intact USP6 protein under the influence of the highly active promoter of the partner gene [5, 44].

In conclusion, MO-like STABC is a benign neoplasm with USP6 rearrangement, characterized with overlapping histological features with $\mathrm{MO}$ and primary bone ABC. Thigh, upper arm, and shoulder are common locations. Peripheral calcification on plain radiograph and CT, perilesional edema, and fluid-fluid levels on MRI are frequently observed radiological features. En-bloc excision is curative when the lesions cause symptoms. Majority of cases show COL1A1-USP6 fusion and herein we have described an additional case with ANGPTL2 gene as a novel fusion partner with USP6.

Acknowledgements This work was supported by the Department of Pathology at Memorial Sloan Kettering Cancer Center Internal Research Fund, and in part by a National Institutes of Health/National Cancer Institute Cancer Center Support Grant under award P30CA008748. The content is solely the responsibility of the authors and does not necessarily represent the official views of the National Institutes of Health. This study was presented in part at the Association of Molecular Pathology 2018 Annual Meeting, November 1, 2018, San Antonio, TX, USA.

\section{Compliance with ethical standards}

Conflict of interest The authors declare that they have no conflict of interest.

Publisher's note Springer Nature remains neutral with regard to jurisdictional claims in published maps and institutional affiliations.

\section{References}

1. Jaffe HL, Lichtenstein L. Solitary unicameral bone cyst: with emphysis on the roentgen picture, the pathologic appearance and the pathogenesis. Arch Surg. 1942;44:1004-25.

2. Panoutsakopoulos G, Pandis N, Kyriazoglou I, Gustafson P, Mertens F, Mandahl N. Recurrent t(16;17)(q22;p13) in aneurysmal bone cysts. Genes Chromosomes Cancer. 1999;26:265-6.

3. Oliveira AM, Hsi BL, Weremowicz S. USP6 (Tre2) fusion oncogenes in aneurysmal bone cyst. Cancer Res. 2004;64:1920-3.

4. Oliveira AM, Perez-Atayde AR, Inwards CY, Medeiros F, Derr V, His BL, et al. USP6 and CDH11 oncogenes identify the neoplastic cell in primary aneurysmal bone cysts and are absent in so-called secondary aneurysmal bone cysts. Am J Pathol. 2004;165:1773-80.

5. Oliveira AM, Perez-Atayde AR, Dal Cin P, Gebhardt MC, Chen CJ, Neff JR, et al. Aneurysmal bone cyst variant translocations upregulate USP6 transcription by promoter swapping with the ZNF9, COL1A1, TRAP150 and OMD genes. Oncogene. 2005;24:3419-26.

6. Panagopoulos I, Mertens F, Löfvenberg R, Mandahl N. Fusion of the COL1A1 and USP6 genes in a benign bone tumor. Cancer Genet Cytogenet. 2008;180:70-3.

7. Guseva NV, Jaber O, Tanas MR, Stence AA, Sompallae R, Schade J, et al. Anchored multiplex PCR for targeted nextgeneration sequencing reveals recurrent and novel USP6 fusions and upregulation of USP6 expression in aneurysmal bone cyst. Genes Chromosomes Cancer. 2017;56:266-77.

8. Šekoranja D, Boštjančič E, Salapura V, Mavčič B, Pižem J. Primary aneurysmal bone cyst with a novel SPARC-USP6 translocation identified by next-generation sequencing. Cancer Genet. 2018;228-9:12-6.

9. Blackburn PR, Davila JI, Jackson RA, Fadra N, Atiq MA, Pitel $\mathrm{BA}$, et al. RNA sequencing identifies a novel USP9X-USP6 promoter swap gene fusion in a primary aneurysmal bone cyst. Genes Chromosomes Cancer. 2019;58:589-94.

10. Sukov WR, Franco MF, Erickson-Johnson M, Chou MM, Unni KK, Wenger DE, et al. Frequency of USP6 rearrangements in myositis ossificans, brown tumor, and cherubism: molecular cytogenetic evidence that a subset of "myositis ossificans-like lesions" are the early phases in the formation of soft-tissue aneurysmal bone cyst. Skelet Radiol. 2008;37:321-7.

11. Bekers EM, Eijkelenboom A, Grünberg K, Roverts RC, de Rooy JW, van der Geest IC, et al. Myositis ossificans-another condition with USP6 rearrangement, providing evidence of a relationship with nodular fasciitis and aneurysmal bone cyst. Ann Diagn Pathol. 2018;34:56-9.

12. Švajdler M, Michal M, Martínek P, Ptáková N, Kinkor Z, Szépe P, et al. Fibro-osseous pseudotumor of digits and myositis ossificans show consistent COL1A1-USP6 rearrangement: a clinicopathological and genetic study of 27 cases. Hum Pathol. 2019;88:39-47.

13. Song W, Suurmeijer AJH, Bollen SM, Cleton-Jansen AM, Bovée JV, Kroon HM. Soft tissue aneurysmal bone cyst: six new cases with imaging details, molecular pathology, and review of the literature. Skelet Radiol. 2019;48:1059-67. 
14. Zheng Z, Liebers M, Zhelyazkova B, Cao Y, Panditi D, Lynch $\mathrm{KD}$, et al. Anchored multiplex PCR for targeted next-generation sequencing. Nat Med. 2014;20:1479-84.

15. Zhu G, Benayed R, Ho C, Mullaney K, Sukhadia P, Rios K, et al. Diagnosis of known sarcoma fusions and novel fusion partners by targeted RNA sequencing with identification of a recurrent ACTB-FOSB fusion in pseudomyogenic hemangioendothelioma. Mod Pathol. 2019;32:609.

16. Ackerman LV. Extra-osseous localized non-neoplastic bone and cartilage formation (so-called myositis ossificans): clinical and pathological confusion with malignant neoplasms. J Bone Jt Surg Am. 1958;40-A:279-98.

17. Meyers C, Lisiecki J, Miller S, Levin A, Fayad L, Ding C, et al. Heterotopic ossification: a comprehensive review. JBMR. 2019;3: e10172.

18. Binnie JF. XIII. Myositis ossificans traumatica. Ann Surg. 1903;38:423-40.

19. Gruca A. Myositis ossificans circumscripta: a clinical and experimental study. Ann Surg. 1925;82:883-919.

20. Amir G, Mogle P, Sucher E. Case report 729. Myositis ossificans and aneurysmal bone cyst. Skelet Radiol. 1992;21:257-9.

21. Rodríguez-Peralto JL, López-Barea F, Sánchez-Herrera S, Atienza M, et al. Primary aneurysmal cyst of soft tissues (extraosseous aneurysmal cyst). Am J Surg Pathol. 1994;18:632-6.

22. Lopez-Barea F, Burgos-Lizaldez E, Rodríguez-Peralto JL, Alvarez-Linera J, Sanchez-Herrera S. Primary aneurysmal cyst of soft tissue: report of a case with ultrastructural and MRI studies. Virchows Arch. 1996;428:125-9.

23. Shannon P, Bedard Y, Bell R, Kandel R. Aneurysmal cyst of soft tissue: report of a case with serial magnetic resonance imaging and biopsy. Hum Pathol. 1997;28:255-7.

24. Nielsen GP, Smith MA, Rosenberg AE. Soft tissue aneurysmal bone cyst. Am J Surg Pathol. 2002;26:6.

25. Wang XL, Gielen JL, Salgado R, Delrue F, De Schepper AMA. Soft tissue aneurysmal bone cyst. Skelet Radiol. 2004;33:477-80.

26. Ajilogba KA, Kaur H, Duncan R, McFarlane JH, Watt AJ. Extraosseous aneurysmal bone cyst in a 12-year-old girl. Pediatr Radiol. 2005;35:1240-2.

27. Ellison DA, Sawyer JR, Parham DM, Nicholas Jr R. Soft-tissue aneurysmal bone cyst: report of a case with $\mathrm{t}(5 ; 17)(\mathrm{q} 33 ; \mathrm{p} 13)$. Pediatr Dev Pathol. 2007;10:46-9.

28. Pietschmann MF, Oliveira AM, Chou MM, Ihrler S, Niederhagen M, Baur-Melnyk A, et al. Aneurysmal Bone cysts of soft tissue represent true neoplasms: a report of two cases. J Bone Jt Surg Am Vol. 2011;93:e45-1-8.

29. Hao Y, Wang L, Yan M, Jin F, Ge S, Dai K. Soft tissue aneurysmal bone cyst in a 10-year-old girl. Oncol Lett. 2012;3:545-8.

30. Baker KS, Gould ES, Patel HB, Hwang SJ. Soft tissue aneurysmal bone cyst: a rare case in a middle aged patient. J Radiol Case Rep. 2015;9. https://doi.org/10.3941/jrcr.v9i1.2157.
31. Jacquot C, Szymanska J, Nemana LJ, Steinbach LS, Horvai AE. Soft-tissue aneurysmal bone cyst with translocation $\mathrm{t}(17 ; 17)(\mathrm{p} 13$; q21) corresponding to COL1A1 and USP6 loci. Skelet Radiol. 2015;44:1695-9.

32. Erickson-Johnson MR, Chou MM, Evers BR, Roth CW, Seys AR, Jin L, et al. Nodular fasciitis: a novel model of transient neoplasia induced by MYH9-USP6 gene fusion. Lab Investig. 2011;91: 1427-33.

33. Patel NR, Chrisinger JSA, Demicco EG, Sarabia SF, Reuther J, Kumar E, et al. USP6 activation in nodular fasciitis by promoterswapping gene fusions. Mod Pathol. 2017;30:1577-88.

34. Carter JM, Wang X, Dong J, Westendorf J, Chou MM, Oliveira AM. USP6 genetic rearrangements in cellular fibroma of tendon sheath. Mod Pathol. 2016;29:865-9.

35. Agaram NP, LeLoarer FV, Zhang L, Hwang S, Athanasian EA, Hameed M, et al. USP6 gene rearrangements occur preferentially in giant cell reparative granulomas of the hands and feet but not in gnathic location. Hum Pathol. 2014;45:1147-52.

36. Yancoskie A, Stojanov I, Fantasia J, Edelman M. USP6 gene rearrangement in cranial fasciitis: a report of three cases. Oral Surg Oral Med Oral Pathol Oral Radiol. 2017;124:e222.

37. Paulson VA, Stojanov IA, Wasman JK, Restrepo T, Cano S, Plunkitt $\mathrm{J}$, et al. Recurrent and novel USP6 fusions in cranial fasciitis identified by targeted RNA sequencing. Mod Pathol. 2019:1-6. https://doi.org/10.1038/s41379-019-0422-6. [Epub ahead of print].

38. Flucke U, Shepard SJ, Bekers EM, Tirabosco R, van Diest PJ, Creytens D, et al. Fibro-osseous pseudotumor of digitsexpanding the spectrum of clonal transient neoplasms harboring USP6 rearrangement. Ann Diagn Pathol. 2018;35:53-5.

39. Online Mendelian Inheritance in Man, OMIM (TM). MIM \#120150. 2018. http://www.ncbi.nlm.nih.gov/omim.

40. Simon MP, Pedeutour F, Sirvent N, Grosgeorge J, Minoletti F, Coindre JM, et al. Deregulation of the platelet-derived growth factor B-chain gene via fusion with collagen gene COL1A1 in dermatofibrosarcoma protuberans and giant-cell fibroblastoma. Nat Genet. 1997;15:95-8.

41. Thorin-Trescases N, Thorin E. Angiopoietin-like-2: a multifaceted protein with physiological and pathophysiological properties. Expert Rev Mol Med. 2014;16:e17.

42. Oliveira AM, Chou MM. The TRE17/USP6 oncogene: a riddle wrapped in a mystery inside an enigma. Front Biosci. 2012;4:321-34.

43. Quick L, Young R, Henrich IC, Wang X, Asmann YW, Oliveira AM, et al. Jak1-STAT3 signals are essential effectors of the USP6/TRE17 oncogene in tumorigenesis. Cancer Res. 2016;76:5337-47.

44. Oliveira AM, Chou MM. USP6-induced neoplasms: the biologic spectrum of aneurysmal bone cyst and nodular fasciitis. Hum Pathol. 2014;45:1-11. 